\title{
Study of the Effect of the Linear Temperature Be- haviour on a Non-Homogeneous Trapezoidal Plate of Parabolically Varying Thickness
}

\author{
Arun Kumar Gupta \\ M. S. College, Saharanpur, U.P., India \\ Pragati Sharma \\ H.C.T.M., Kaithal, Haryana, India
}

(Received 8 February 2012; revised 16 October 2012; accepted 6 February 2013)

An analysis is presented for studying the effect of the linear temperature behaviour on the transverse vibration of a non-homogeneous trapezoidal plate of varying thickness on the basis of classical plate theory. The nonhomogeneity of the plate material is assumed to arise due to the variation in density, which is assumed to vary parabolically. The thickness of the plate also varies parabolically. A two-term deflection function is performed to solve the equation of motions using the Rayleigh-Ritz method. The frequency equation is derived when two edges of the plate are simply supported and two are clamped, which is called clamped simply-supported clamped simplysupported. Effects of the non-homogeneity with other plate parameters-such as aspect ratio, taper constant, and thermal gradient on the first two modes of vibration - have been analysed. Results are presented in graphical form.

\section{NOMENCLATURE}

$\begin{array}{ll}\omega & \text { Angular frequency } \\ a & \text { Length of the plate } \\ b & \text { Width of the plate at left edge } \\ c & \text { Width of the plate at right edge } \\ x & \text { Longitudinal coordinate } \\ y & \text { Vertical coordinate } \\ \alpha_{1} & \text { Non-homogeneity constant } \\ h & \text { Plate thickness } \\ E & \text { Young's modulus } \\ v & \text { Poisson's ratio } \\ \tau & \text { Temperature distribution } \\ \xi & \text { Non-dimensional coordinate }=\frac{x}{a} \\ \eta & \text { Non-dimensional coordinate }=\frac{y}{b} \\ T & \text { Kinetic energy } \\ V & \text { Strain energy } \\ \lambda & \text { Frequency parameter } \\ z & \text { Transverse coordinate } \\ w & \text { Deflection function } \\ D(\xi) & \text { Flexural rigidity } \\ \beta & \text { Thermal gradient } \\ \alpha & \text { Taper constant }\end{array}$

\section{INTRODUCTION}

Plates of varying shape and thickness are found in many engineering structures and are used extensively in machine design, nuclear reactor technology, naval structures, and acoustical components. The practical importance of dynamic analysis, in addition to the classical static analysis and the numerical evaluation of the vibrational characteristics of structural elements such as plates, has become an important part of the design process.

Vibration phenomenon, common in mechanical devices and structures, is undesirable in many cases, such as machine tools. But this phenomenon is not always unwanted; for example, vibration is needed in the operation of vibration screens. Over time, engineers have become increasingly conscious of the importance of the elastic behaviour of plates as well as the natural frequencies and mode shapes of the plates, which, from a technical point of view, is indispensable information. For reasons of both practical and academic interests, numerous publications concerned with the vibration of plates have been published.

Gupta and Sharma estimated the effect of thermal gradient on transverse vibrations of an orthotropic trapezoidal plate of linearly varying thickness. ${ }^{1}$ Gurses et al. did the analysis of shear deformable laminated composite trapezoidal plates. ${ }^{2}$ Kang and Lee gave the free vibration analysis of an unsymmetric trapezoidal membrane. ${ }^{3}$ Khani and Aziz observed the thermal analysis of a longitudinal trapezoidal fin with temperaturedependent thermal conductivity and the heat transfer coefficient. $^{4}$ Leung et al. calculated the free vibration of laminated composite plates subjected to in-plane stresses using the trapezoidal $p$-element. ${ }^{5}$ Liew and Lim discussed a global continuum Ritz formulation for flexural vibration of pre-twisted trapezoidal plates with one edge built in. ${ }^{6}$ Liew used a hybrid energy approach for the vibrational modelling of laminated trapezoidal plates with point supports. ${ }^{7}$ Liew also evaluated the vibration of symmetrically laminated cantilever trapezoidal composite plates. ${ }^{8}$ Maruyama et al. carried out an experimental study of the free vibration of clamped trapezoidal plates. ${ }^{9}$ 